\title{
First-principles calculations on elastic and entropy properties in FeRh alloys
}

\author{
Wangqiang $\mathrm{He}^{1 *}$, Houbing Huang ${ }^{1 *}$, Xingqiao $\mathrm{Ma}^{1 \#}$
}

First-principles calculations were employed to investigate elastic and entropy properties of $B 2$ ferromagnetic and antiferromagnetic FeRh phases. We calculated lattice parameters, elastic constants, isotropic moduli like bulk modulus $B$, shear modulus $G$, Young's modulus $E$, and Poisson's ratio of two phases and compared these with other calculations and experiments. At finite temperatures, the values of vibrational (from both Debye model and phonon dispersion) and thermal electronic contributions to the total entropy change between the two phases are calculated about $-50 \mathrm{~J} / \mathrm{kg} / \mathrm{K}$ and $7.8 \mathrm{~J} / \mathrm{kg} / \mathrm{K}$ comparable to the experimental results $(-33 \pm 9 \mathrm{~J} / \mathrm{kg} / \mathrm{K}$ and $8 \pm 1 \mathrm{~J} / \mathrm{kg} / \mathrm{K})$.

Keywords: Elastic constants, Isotropic moduli, Pugh ratio, Entropy change, First-principles calculations 
Recently, magnetocaloric effect (MCE) in LaFeSi [1], FeRh [2-4], and NiCoMnIn Heusler [5, 6] alloys has received much attention because it's potential application in magnetic refrigeration technology an alternative technology to the current gas-compression method. The MCE is characterized by two parameters: the adiabatic temperature change $\Delta T$ and the isothermal entropy change $\Delta S$. The total entropy change $\Delta S_{\text {tot }}$ usually can be written as the sum of i.e.: $\Delta S_{\text {tot }}=\Delta S_{\text {ele }}+\Delta S_{\text {lat }}+\Delta S_{\text {mag }}$ the entropy change of the lattice $\Delta S_{\text {lat }}$, of the magnetic $\Delta S_{\text {mag }}$ and of the thermal electronic system $\Delta S_{\text {ele }}$. The study on the separation of these contributions to the MCE is of great importance to understand this giant MCE [6]. By direct experimental measurement of the MCE in NiCoMnIn alloys, Kihara et al. [6] showed that $\Delta S_{\text {ele }}$ is negligibly small whereas $\Delta S_{\text {lat }}$ plays the dominant role in the MCE. Gruner et al. [1] demonstrated that $\Delta S_{\text {lat }}$ contributes cooperatively with the magnetic and thermal electronic entropy changes in LaFeSi by nuclear resonant inelastic X-ray scattering and first-principles calculations. Cooke et al. [7] reported $\Delta S_{\text {lat }}=-33 \mathrm{~J} / \mathrm{kg} / \mathrm{K}$ and $\Delta S_{\text {ele }}=8 \mathrm{~J} / \mathrm{kg} / \mathrm{K}$ by low-temperature specific heat and photoemission measurements in FeRh alloys, which is promising material for the magnetic refrigeration because of the highest $\Delta T=12.9 \mathrm{~K}$ of all known materials [8].

However, recent first-principles calculations underestimated the difference in entropy of lattice and thermal electronic which was $11.7 \mathrm{~J} / \mathrm{kg} / \mathrm{K}$ [9] (experimental value $-25 \mathrm{~J} / \mathrm{kg} / \mathrm{K}$ [7]). Besides, the first-principles determination of isotropic moduli which is important for application and thermodynamical parameters like Debye temperatures are lacking in the literature. In this paper, we firstly determined the lattice parameters, elastic constants, and isotropic moduli of the two phases. Then we obtained the values of lattice vibrational (from both Debye model and phonon dispersion) and thermal electronic contributions to the total entropy change.

\section{Computational details}

First-principles calculations were carried out with the Vienna ab-initio simulation package (VASP) code $[10,11]$ and the projector augmented wave (PAW) as well as the Perdew-Burke-Ernzerhof (PBE) formulation of the generalized gradient approximation (GGA) [12]. The kinetic energy cut-off of $650 \mathrm{eV}$ was used for plane-wave set, and the Brillouin zone (BZ) was sampled with special k-points of an 


\section{Results and discussion}

As previous theoretical publication showed [15], the B2 FeRh exhibits two magnetic structures, ferromagnetic (FM) and $G$-type antiferromagnetic (AFM) ordering. The structural properties are investigated by the 4-th order Murnaghan Equation of State (EOS) equation (1).

$$
E(\mathrm{~V})=\frac{B_{0}}{B^{\prime}\left(B^{\prime}-1\right)}\left[\left(\frac{V_{0}}{V}\right)+B^{\prime}\left(1-\frac{V_{0}}{V}\right)-1\right]+E_{0}
$$

The predicted equilibrium properties are given in Table I, including lattice parameter $a_{0}$, bulk modulus $B$, and local magnetic moment. We find the predicted lattice parameter of FM phase is $3.017 \AA$ larger than that of AFM phase $3.000 \AA$ and the volume change is about $1.7 \%$. The local Fe magnetic moment remains large and essentially constant around $3.14 \mu_{B}$ in two phases (smaller than experimental value $\left.3.30 \mu_{B}[18]\right)$. Meanwhile Rh atom possesses $1.0 \mu_{B}$ in FM phase and loss of its moment in AFM phase. As shown in Table I, the elastic constants of the two phases are obtained with strains $( \pm 0.007)$ by strain-stress method [19] and fulfill the mechanical stability criteria for cubic structures:

$$
C_{11}-C_{12}>0, C_{11}>0, C_{33}>0, C_{11}+2 C_{12}>0
$$

Table I also lists the isotropic moduli for two phases like bulk modulus $B$, shear modulus $G$, Young's modulus $E$, and Poisson's ratio $v$ and experimental values are collected for comparison. The bulk and shear modulus are estimated by the Voigt-Reuss-Hill approach. The Young modulus and Poisson's ratio are predicted by Hill approach. It should be noted that the calculated $B$ of two phases from elastic constants (EC) agree well with those directly from the fitting of (EOS) listed in Table I, which further demonstrates the good accuracy of our elastic constants calculations. Also our calculated $B$ is consistent 
with the recent work [15] and 1.4 times larger than the experiment [7]. The large difference can be explained by following two reasons: (1) Present calculations did not consider the effect of thermal expansion effect (2) the amorphous and off-stoichiometric nature of the experimental material. In Table I $E$ decrease by as much as $20 \%$ from FM to AFM phase as well as an increment of $15 \%$ in $v$ and both agree well with the experiment [7]. The AFM phase has a slightly higher $B$ than FM phase while the FM phase has a higher $G$ and $E$ than AFM phase. According to Pugh criteria [20], $B / G$ ratio of 1.75 separates ductile and brittle materials. The two phases show ductile property especially AFM phase since their Pugh ratio $B / \mathrm{G}>1.75$.

Then we discuss entropy change during AFM-FM transition. Figure 1 shows the phonon dispersion and vibrational density of states (VDOS) for two phases. It is observed that FM phase is dynamical stable since all phonon modes have real values in the whole Brillouin zone. However, the AFM phase shows imaginary frequency at $\mathrm{X}$ and $\mathrm{K}$ points which means AFM is dynamically unstable [15]. This lattice instability in the AFM phase leads to the discovery of a stable monoclinic or metastable face-centered-cubic-like structure [9, 15]. By the harmonic approximation $\Delta S_{\text {lat }}=-10.8 \mathrm{~J} / \mathrm{kg} / \mathrm{K}$ $(-11.7 \mathrm{~J} / \mathrm{kg} / \mathrm{K}[9])$ is much smaller than the experimental results $-39 \mathrm{~J} / \mathrm{kg} / \mathrm{K}$. This discrepancy is on account that AFM phase is stable below the transition temperature in the experiment. Here we applied the Hubbard- $U$ correction ( $U=1 \mathrm{eV}$ ) to the Fe $d$ orbitals to stabilize AFM phase [15]. In Figure 1(a) the phonon dispersion of FM phase with $\mathrm{DFT}+U$ is similar with PBE calculations. However, that of AFM phase shows no imaginary frequency at $\mathrm{X}$ or $\mathrm{K}$ points with $\mathrm{DFT}+U$ calculations and it gives Fe magnetic moment equal to $3.35 \mu_{B}$ close to experimental value $3.30 \mu_{B}$. Figure 2 shows $\Delta S_{\text {lat }}=-50 \mathrm{~J} / \mathrm{kg} / \mathrm{K}$ with the stable AFM phase. Besides, $\Delta S_{\text {lat }}$ is estimated about $-50 \mathrm{~J} / \mathrm{kg} / \mathrm{K}$ by Debye theory [21] just the same as lattice dynamics predicted in Figure 2.

Next, we investigate the thermal electronic entropy $S_{e l}$ with the form $[22,23]$

$$
S^{e l}=-k_{B} \int n(\varepsilon)[f \ln f+(1-f) \ln (1-f)] d \varepsilon
$$

Where $n(\varepsilon)$ is the total electronic density of states (DOS) with $f$ being the Fermi distribution. $\Delta S_{\text {ele }}=S_{F M}-S_{A F M}$ by equation (4) is estimated about $7.8 \mathrm{~J} / \mathrm{kg} / \mathrm{K}(8 \mathrm{~J} / \mathrm{kg} / \mathrm{K}$ expt.[7]) at $370 \mathrm{~K}$ in 


\section{Conclusions}

In summary, the DFT calculations are used to investigate the elastic and entropy properties of $B 2 \mathrm{FeRh}$ in FM and AFM phases. The calculated lattice parameters, elastic constants, bulk modulus, shear modulus, Young's modulus, and Poisson's ratio of the two phases agree with experimental results. The Pugh ratio indicates the two phases especially AFM exhibits ductile property. We obtain $\Delta S_{\text {lat }}=-50 \mathrm{~J} / \mathrm{kg} / \mathrm{K}$ using first-principles phonon calculations as well as Debye model and $\Delta S_{\text {ele }}=7.8 \mathrm{~J} / \mathrm{kg} / \mathrm{K}$ by the DFT calculated DOS which is insufficient to drive AFM-FM transition in FeRh alloys. The discrepancy between the calculated $\gamma_{F M}=32.6 \mathrm{~mJ} / \mathrm{kg} / \mathrm{K}^{2}$ and experimental value $53-62.5 \mathrm{~mJ} / \mathrm{kg} / \mathrm{K}^{2}$ may be due to the entropy contribution of the magnon excitations in FM phase. 


\section{Acknowledgements}

This work was supported by National Science Foundation of China under Grant No. 11504020 and No. 1174030.

\section{References}

[1] Gruner ME, Keune W, Roldan Cuenya B, Weis C, Landers J, Makarov SI, et al. Element-resolved thermodynamics of magnetocaloric $\mathrm{LaFe}_{(13-\mathrm{x})} \mathrm{Si}_{(\mathrm{x})}$. Phys Rev Lett. 2015;114:057202.

[2] Chirkova A, Skokov KP, Schultz L, Baranov NV, Gutfleisch O, Woodcock TG. Giant adiabatic temperature change in FeRh alloys evidenced by direct measurements under cyclic conditions. Acta Mater. 2016;106:15-21.

[3] Zverev VI, Saletsky AM, Gimaev RR, Tishin AM, Miyanaga T, Staunton JB. Influence of structural defects on the magnetocaloric effect in the vicinity of the first order magnetic transition in $\mathrm{Fe}_{50.4} \mathrm{Rh}_{49.6}$. Appl Phys Lett. 2016;108:192405.

[4] Stern-Taulats E, Gràcia-Condal A, Planes A, Lloveras P, Barrio M, Tamarit J-L, et al. Reversible adiabatic temperature changes at the magnetocaloric and barocaloric effects in $\mathrm{Fe}_{49} \mathrm{Rh}_{51}$. Appl Phys Lett. 2015;107:152409.

[5] Gottschall T, Skokov KP, Benke D, Gruner ME, Gutfleisch O. Contradictory role of the magnetic contribution in inverse magnetocaloric Heusler materials. Phys Rev B. 2016;93:184431.

[6] Kihara T, Xu X, Ito W, Kainuma R, Tokunaga M. Direct measurements of inverse magnetocaloric effects in metamagnetic shape-memory alloy NiCoMnIn. Phys Rev B. 2014;90:214409.

[7] Cooke DW, Hellman F, Baldasseroni C, Bordel C, Moyerman S, Fullerton EE. Thermodynamic measurements of Fe-Rh alloys. Phys Rev Lett. 2012;109:255901.

[8] Nikitin SA, Myalikgulyev G, Tishin AM, Annaorazov MP, Asatryan KA, Tyurin AL. The magnetocaloric effect in $\mathrm{Fe}_{49} \mathrm{Rh}_{51}$ compound. Phys Lett A. 1990;148:363.

[9] Wolloch M, Gruner ME, Keune W, Mohn P, Redinger J, Hofer F, et al. Impact of lattice dynamics on the phase stability of metamagnetic FeRh: Bulk and thin films. Phys Rev B. 2016;94:174435.

[10] Kresse G, Hafner J. Ab initio molecular dynamics for liquid metals. Phys Rev B. 1993;47:558.

[11] Kresse G, Furthmüller J. Efficient iterative schemes for ab initio total-energy calculations using a plane-wave basis set. Phys Rev B. 1996;54:11169.

[12] Perdew JP, Burke K, Ernzerhof M. Generalized Gradient Approximation Made Simple. Phys Rev Lett. 1996;77:3865.

[13] Methfessel M, Paxton A. High-precision sampling for Brillouin-zone integration in metals. Phys Rev B. 1989;40:3616.

[14] Dudarev SL, Botton GA, Savrasov SY, Humphreys CJ, Sutton AP. Electron-energy-loss spectra and the structural stability of nickel oxide: An LSDA+U study. Phys Rev B. 1998;57:1505.

[15] Aschauer U, Braddell R, Brechbühl SA, Derlet PM, Spaldin NA. Strain-induced structural instability in FeRh. Phys Rev B. 2016;94:014109. 
[16] Togo A, Oba F, Tanaka I. First-principles calculations of the ferroelastic transition between rutile-type and $\mathrm{CaCl}_{2}$-type $\mathrm{SiO}_{2}$ at high pressures. Phys Rev B. 2008;78:134106.

[17] Togo A, Tanaka I. First principles phonon calculations in materials science. Scripta Mater. 2015;108:1-5.

[18] Shirane G, Nathans R, Chen CW. Magnetic Moments and Unpaired Spin Densities in the Fe-Rh Alloys. Physical Review. 1964;134:A1547-A53.

[19] Shang S, Wang Y, Liu Z-K. First-principles elastic constants of $\alpha$ - and $\theta-\mathrm{Al}_{2} \mathrm{O}_{3}$. Appl Phys Lett. 2007;90:101909.

[20] Pugh SF. XCII. Relations between the elastic moduli and the plastic properties of polycrystalline pure metals. The London, Edinburgh, and Dublin Philosophical Magazine and Journal of Science. 1954;45:823-43.

[21] Moruzzi VL, Janak JF, Schwarz K. Calculated thermal properties of metals. Phys Rev B. 1988;37:790.

[22] Wang Y, Liu ZK, Chen LQ. Thermodynamic properties of $\mathrm{Al}, \mathrm{Ni}, \mathrm{NiAl}$, and Ni3Al from first-principles calculations. Acta Mater. 2004;52:2665-71.

[23] Wang Y, Hector L, Zhang H, Shang S, Chen L, Liu Z. Thermodynamics of the Ce $\gamma-\alpha$ transition: Density-functional study. Phys Rev B. 2008;78:104113.

[24] Tu P. Mechanism for the First-Order Magnetic Transition in the FeRh System. J Appl Phys. 1969;40:1368.

[25] Gruner ME, Hoffmann E, Entel P. Instability of the rhodium magnetic moment as the origin of the metamagnetic phase transition in $\alpha-F e R h$. Phys Rev B. 2003;67:064415.

[26] Gu RY, Antropov VP. Dominance of the spin-wave contribution to the magnetic phase transition in FeRh. Phys Rev B. 2005;72:012403.

\section{Figure captions}

Figure 1. Phonon band structure and vibrational density of states (VDOS) for (a) FM (b) AFM FeRh alloys computed with PBE and DFT $+U$.

Figure 2. Lattice and thermal electric contribution to entropy change between FM and AFM phase vs temperature. Total entropy change is the sum of the lattice and thermal electriconic contribution. 

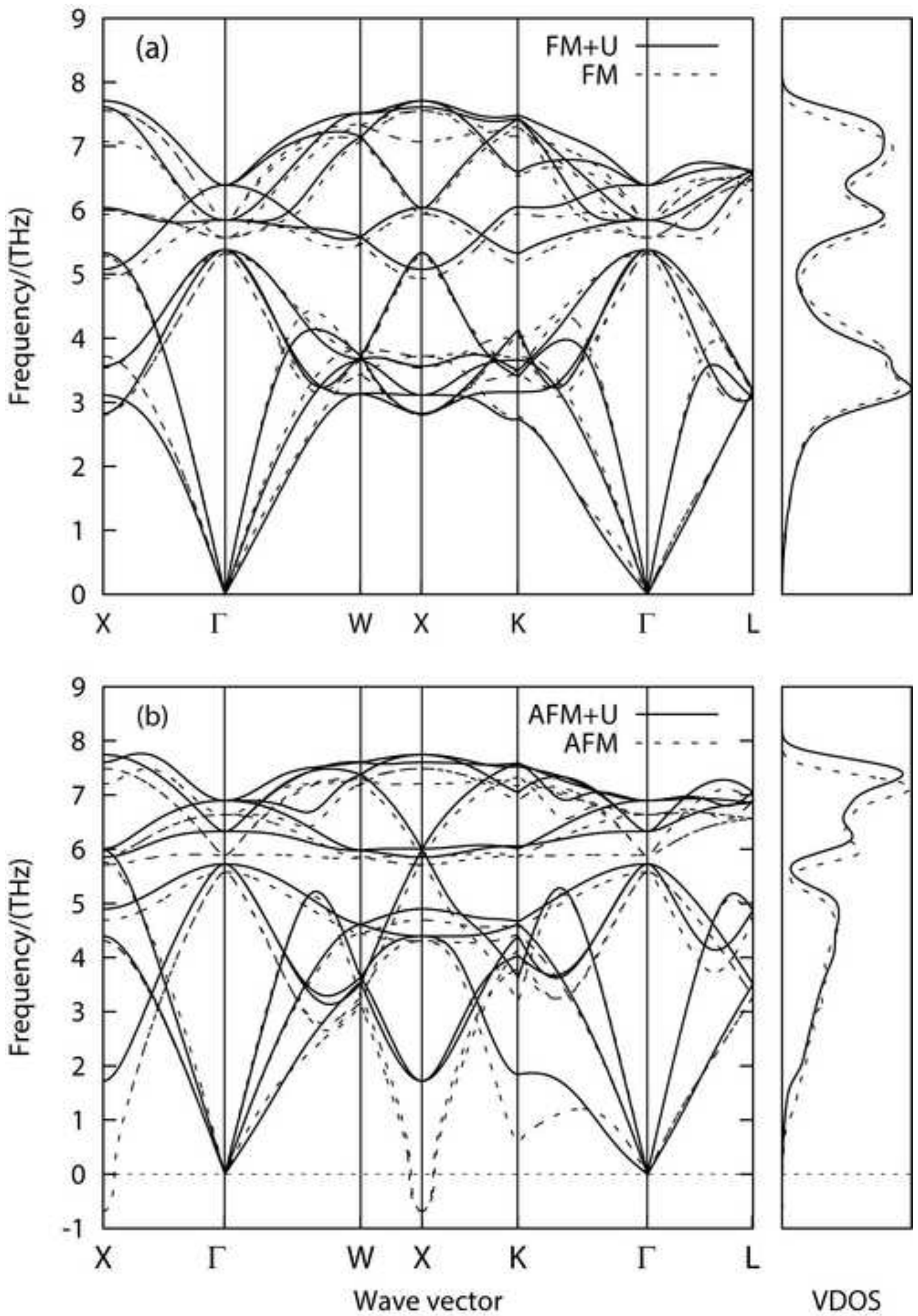


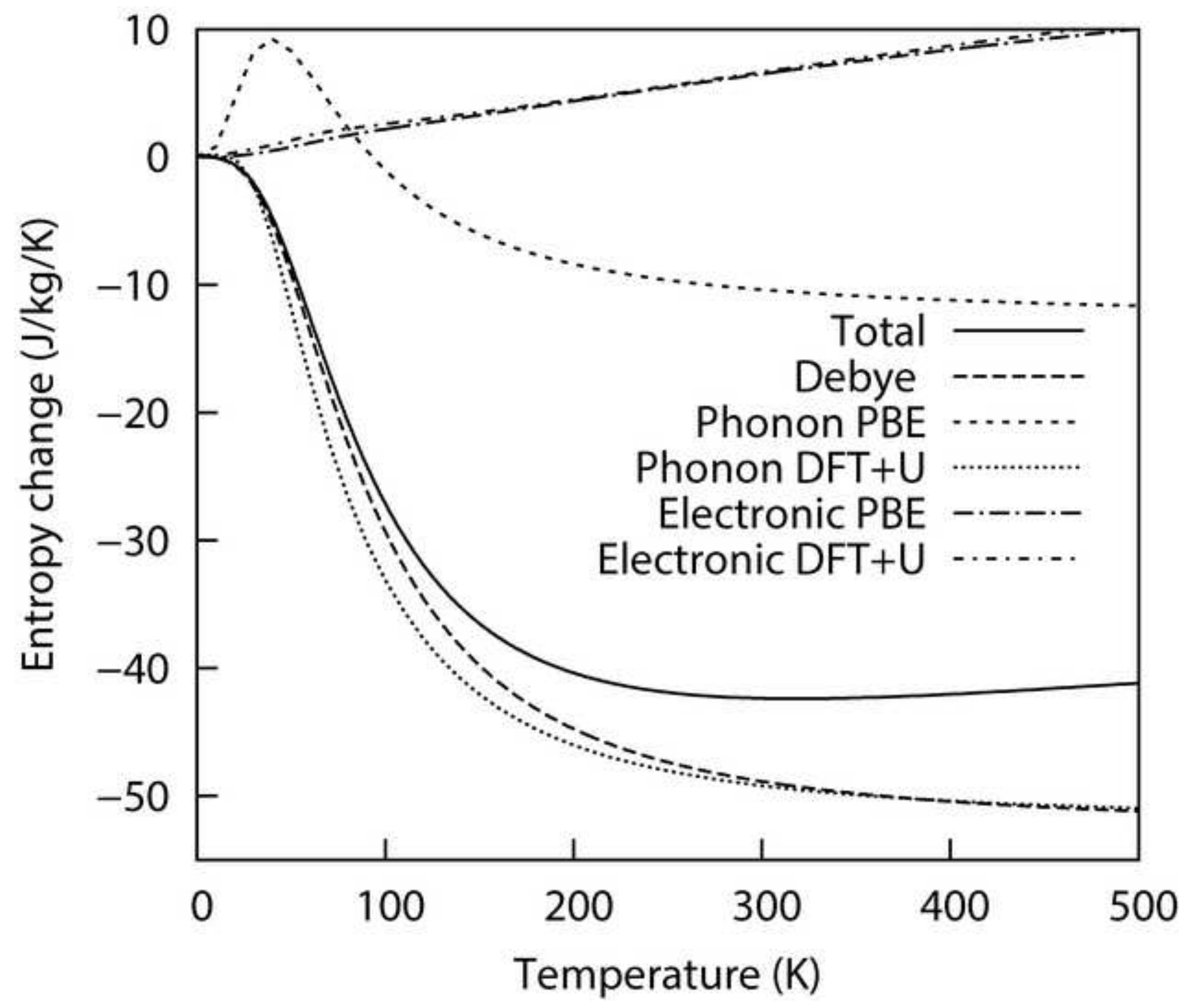


Table I. The calculated equilibrium lattice constant $a_{0}$ in $\AA$, local magnetic moment $m$ in $\mu_{B}$, bulk modulus $B$ in GPa, Debye temperature $\Theta_{D}$ in K, elastic constants and isotropic modulus in GPa, Poisson's ratio $v$ and Pugh ratio of FeRh alloys. ${ }^{*}$ calculated from $\mathrm{DFT}+U$.

\begin{tabular}{|c|c|c|c|c|c|c|c|c|c|c|c|c|c|c|}
\hline \multirow[t]{2}{*}{ Phase } & & $a_{0}$ & \multicolumn{2}{|l|}{$m$} & \multirow{2}{*}{$\begin{array}{c}B \\
\text { EOS }\end{array}$} & \multirow[t]{2}{*}{$\Theta_{D}$} & \multirow[t]{2}{*}{$C_{11}$} & \multirow[t]{2}{*}{$C_{12}$} & \multirow[t]{2}{*}{$C_{44}$} & \multirow{2}{*}{$\begin{array}{c}B \\
\mathrm{EC}\end{array}$} & \multirow[t]{2}{*}{$G$} & \multirow[t]{2}{*}{ E } & \multirow[t]{2}{*}{$v$} & \multirow[t]{2}{*}{$B / G$} \\
\hline & & & $\mathrm{Fe}$ & $\mathrm{Rh}$ & & & & & & & & & & \\
\hline \multirow[t]{3}{*}{ FM } & This work & 3.017 & $3.183 .33^{*}$ & 1.04 & 195.3 & 396 & 278.3 & 179.4 & 110.2 & 194.9 & 78.1 & 206.5 & 0.32 & 2.49 \\
\hline & Ref.[7] & & & & & 393 & & & & 133 & & 197 & 0.25 & \\
\hline & Ref.[15] & 3.018 & 3.21 & 1.05 & & & 251.5 & 164.2 & 109.8 & 193.3 & & & & \\
\hline \multirow[t]{3}{*}{ AFM } & This work & 3.000 & $3.143 .35^{*}$ & 0 & 198.2 & 335 & 218.7 & 188.5 & 119.8 & 198.5 & 54.8 & 150.6 & 0.37 & 3.62 \\
\hline & Ref.[7] & & & & & 340 & & & & 142 & & 153 & 0.32 & \\
\hline & Ref.[15] & 3.002 & 3.15 & 0 & & & 225.4 & 181.2 & 119.9 & 195.9 & & & & \\
\hline
\end{tabular}

